\title{
A NOTE ON COMMUTATORS AND SINGULAR INTEGRALS ${ }^{1}$
}

\author{
PAUL S. MUHLY
}

\begin{abstract}
A new approach to the analysis of a certain commutator equation is presented.
\end{abstract}

1. Introduction. In this note we apply a technique due to Douglas [2] to analyze the solutions to the operator equation

$$
H X-X H=i R^{2}
$$

where $H$ is a (possibly unbounded) selfadjoint operator on a (complex) Hilbert space $\mathcal{K}_{,}^{2} R$ is a bounded nonnegative operator on $\mathcal{H}$, and where the unknown $X$ is a bounded selfadjoint operator on $\mathcal{F}_{0}$ Our analysis will provide a new and elementary proof of a well-known theorem which is due, in various forms, to Xa Dao-xeng [8], Pincus [5], and Kato [4]. Our approach has certain points of contact with Kato's but differs from his in that instead of making a detailed analysis of the resolvent of $H$, we use a basic integral which appears in perturbation theory, and which he used too, to prove a theorem of Putnam [6] by exhibiting an explicit unitary equivalence between $H$ and multiplication by $x$ on a direct integral based on Lebesgue measure on $\mathbf{R}$. This unitary equivalence is then easily seen to be one also between $X$ and an explicit singular integral operator on the direct integral.

Since $H$ may be unbounded, what is meant by a solution to equation (1) is open to interpretation. Therefore we specify now that by a solution to equation (1) we shall mean a bounded selfadjoint operator $X$ such that $X \operatorname{Dom}(H) \subseteq \operatorname{Dom}(H)$ and such that $(H X-X H) f=i R^{2} f$ for all $f$ $\in \operatorname{Dom}(H)$.

2. The solution. We shall write $U_{t}=e^{i t H}, t \in \mathbf{R}$.

Recall that $f$ belongs to $\operatorname{Dom}(H)$ precisely when

$$
\frac{d}{d t} U_{t} f \stackrel{\text { def }}{=} \lim _{h \rightarrow 0} \frac{U_{t+h} f-U_{t} f}{h}
$$

exists, and that for such $f,(d / d t) U_{t} f=i H U_{t} f=i U_{t} H f$. Consequently, if $T$ is any bounded linear transformation on $\mathcal{H}$ which maps $\operatorname{Dom}(H)$ into $\operatorname{Dom}(H)$,

\footnotetext{
Received by the editors October 23, 1974 and, in revised form, May 13, 1975.

AMS (MOS) subject classifications (1970). Primary 47B47, 47G05; Secondary 45E10, 47A55.

Key words and phrases. Commutator, singular integral, perturbation theory.

1 This work was supported in part by a grant from the National Science Foundation.

2 We assume $\mathcal{K}$ is separable so that at one point we may apply direct integral theory. However, as the reader may readily verify for himself, this is not really necessary for the rest of the paper.
} 
then

$$
\frac{d}{d t} U_{t} T U_{t}^{*} f \stackrel{\text { def }}{=} \lim _{h \rightarrow 0} \frac{U_{t+h} T U_{t+h}^{*} f-U_{t} T U_{t}^{*} f}{h}
$$

exists for all $f \in \operatorname{Dom}(H)$ and, of course, is $U_{t}(i(H T-T H)) U_{t}^{*}$. Thus a bounded selfadjoint operator $X$ satisfies equation (1) if and only if $X \operatorname{Dom}(H)$ $\subseteq \operatorname{Dom}(H)$ and $(d / d t) U_{t} X U_{t}^{*} f=-U_{t} R^{2} U_{t}^{*} f$ for all $f \in \operatorname{Dom}(H)$.

LEMMA 2.1. There is at most one solution $Q$ to equation (1) which satisfies the equation

$$
\lim _{t \rightarrow \infty} U_{t} Q U_{t}^{*}=0
$$

in the strong operator topology. Moreover, if a solution to equation (1) exists, then a solution $Q$ satisfying equation (2) also exists, is nonnegative, and every solution $X$ to equation (1) may be written as $X=Q+A$ where $A$ commutes with $H .^{3}$

Proof. Suppose $Q_{1}$ and $Q_{2}$ are solutions to equation (1) satisfying equation (2). Then $Q_{1}-Q_{2}$ commutes with $\left\{U_{t}\right\}_{t \in \mathbf{R}}$ and by equation (2) must be zero. Let $X$ be a solution to equation (1). Then since $(d / d t) U_{t} X U_{t}^{*} f=-U_{t} R^{2} U_{t}^{*} f$, $f \in \operatorname{Dom}(H),\left\{U_{t} X U_{t}^{*}\right\}_{t \in \mathbf{R}}$ is a decreasing family of bounded selfadjoint operators. Since this family is clearly bounded below, $\lim _{t \rightarrow \infty} U_{t} X U_{t}^{*}$ exists in the strong operator topology and defines a bounded selfadjoint operator $A$ which clearly commutes with $\left\{U_{t}\right\}_{t \in \mathbf{R}}$. Hence $A$ commutes with $H$ and so $X-A$ is a nonnegative solution to equation (1) which satisfies equation (2). The proof is complete.

Definition 2.2. If equation (1) has a solution, then the unique solution satisfying equation (2) is called the principal solution of equation (1).

We let $\mathscr{D}$ denote the closure of the range of $R$ and we let $\mathbf{L}_{\mathscr{D}}^{2}(\mathbf{R})$ denote the collection of all weakly measurable $\Phi$-valued functions on $\mathbf{R}$ which are norm square integrable with respect to Lebesgue measure on $\mathbf{R}$. The subspace of $\mathbf{L}_{\text {D }}^{2}(\mathbf{R})$ consisting of those functions which vanish a.e. on $(-\infty, 0)$ will be denoted by $\mathbf{L}_{\mathscr{D}}^{2}([0, \infty))$ and the projection from $\mathbf{L}_{\mathscr{D}}^{2}(\mathbf{R})$ onto $\mathbf{L}_{\mathscr{D}}^{2}([0, \infty))$ will be denoted by $P$. Finally, $\left\{S_{t}\right\}_{t \in \mathbf{R}}$ will denote the unitary group defined on $\mathbf{L}_{\mathfrak{P}}^{2}(\mathbf{R})$ by the formula $\left(S_{t} f\right)(x)=f(x-t), f \in \mathbf{L}_{\mathscr{D}}^{2}(\mathbf{R}), t \in \mathbf{R}$, and $\left\{\tilde{S}_{t}\right\}_{t \geq 0}$ will denote the semigroup of isometries defined on $\mathbf{L}_{\mathscr{D}}^{2}([0, \infty))$ by restricting $S_{t}$, $t \geq 0$, to $\mathbf{L}_{\mathscr{D}}^{2}([0, \infty))$; i.e., $\tilde{S}_{t}=S_{t} \mid \mathbf{L}_{\mathscr{D}}^{2}([0, \infty)), t \geq 0$, where the vertical bar denotes restriction.

THEOREM I. Suppose equation (1) has a solution and let $Q$ be its principal one. Then there is a bounded linear transformation $C$ from $\mathcal{H}$ to $\mathbf{L}_{\mathscr{D}}^{2}(\mathbf{R})$ such that $C U_{t}^{*}=S_{t}^{*} C$ for all $t \in \mathbf{R}$ and such that $Q=C^{*} P C$.

Proof. First note that from the discussion preceding Lemma 2.1, we may infer that for $f \in \operatorname{Dom}(H)$,

$$
\begin{aligned}
\int_{0}^{\infty} U_{t} R^{2} U_{t}^{*} f d t & =\lim _{T \rightarrow \infty}-\int_{0}^{T} \frac{d}{d t} U_{t} Q U_{t}^{*} f d t \\
& =Q f-\lim _{T \rightarrow \infty} U_{T} Q U_{T}^{*} f=Q f .
\end{aligned}
$$

${ }^{3}$ This means that $A \operatorname{Dom}(H) \subseteq \operatorname{Dom}(H)$ and $A H f=H A f, f \in \operatorname{Dom}(H)$. Since $\operatorname{Dom}(H)$ $=\left\{f \mid(d / d t) U_{t} f\right.$ exists $\}$ it is clear that $A$ commutes with $H$ if and only if $A$ commutes with $\left\{U_{t}\right\}_{t \in \mathbf{R}}$. 
Hence, since $\operatorname{Dom}(H)$ is dense and $Q$ is bounded, $\int_{0}^{\infty} U_{t} R^{2} U_{t}^{*} d t$ converges as an improper integral in the strong operator topology to $Q$. For $f \in \mathcal{H}$, we define the $\Phi$-valued function $\tilde{C} f$ on $[0, \infty)$ by the formula $(\tilde{C} f)(t)=R U_{t}^{*} f$. This function is in $\mathbf{L}_{\mathscr{D}}^{2}([0, \infty))$ because

$$
\int_{0}^{\infty}\|(\tilde{C} f)(t)\|^{2} d t=\int_{0}^{\infty}\left(U_{t} R^{2} U_{t}^{*} f, f\right) d t=(Q f, f)=\left\|Q^{1 / 2} f\right\|^{2},
$$

and so $\tilde{C}$ is a bounded linear transformation from $\mathcal{H}$ to $\mathbf{L}_{\mathscr{D}}^{2}([0, \infty))$ satisfying the equation $Q=\tilde{C}^{*} \tilde{C}$. Clearly $\tilde{C} U_{t}^{*}=\tilde{S}_{t}^{*} \tilde{C}$ for all $t \geq 0$. Since the minimal unitary extension of the semigroup of isometries $\left\{\tilde{S}_{t}\right\}_{t \geq 0}$ is $\left\{S_{t}\right\}_{t \in \mathbf{R}}$ and since, $\left\{U_{t}\right\}_{t>0}$ is a semigroup of unitary operators, a well-known theorem (see [3]) implies that there is a unique bounded linear transformation $C$ mapping $\mathcal{H}$ to $\mathbf{L}_{\mathscr{D}}^{2}(\mathbf{R})$ such that $C^{*}$ extends $\tilde{C}^{*}$ and such that $C U_{t}^{*}=S_{t}^{*} C$ for all $t \in \mathbf{R}$. Hence $\tilde{C}=P C$ and $Q=C^{*} P C$.

3. Applications. In this section we use Theorem I to obtain the results of $\mathrm{Xa}$ Dao-xeng, Pincus, and Kato promised in the introduction. Along the way we present a new proof of a theorem of Putnam.

We continue to use the notation established in $\S 2$; and if $A$ is a bounded linear transformation (possibly between different Hilbert spaces), we will write $\mathfrak{\Im}(A)$ for the initial space of $A$; i.e., $\mathfrak{\Im}(A)=(\operatorname{ker}(A))^{\perp}$.

Lemma 3.1. Suppose equation (1) has a solution and let $Q$ be its principal one. Then $\Im(Q)$ is the smallest subspace of $\mathcal{H}$ containing the range of $R^{2}$ and invariant under $\left\{U_{t}\right\}_{t \geq 0}$.

Proof. Theorem I could be used for part of the proof, but it is just as easy to proceed without it. Since $U_{t} Q U_{t}^{*} \leq Q, t \geq 0$, it follows that $\operatorname{ker}(Q)$ is invariant under $\left\{U_{t}^{*}\right\}_{t \geq 0}$. Since

$$
R^{2} f=\lim _{t \rightarrow 0+} \frac{U_{t} Q U_{t}^{*} f-Q f}{t}
$$

for all $f$ such that the limit exists, it follows that $\operatorname{ker}(Q) \subseteq \operatorname{ker}\left(R^{2}\right)$. Upon taking orthogonal complements, we find that the range of $R^{2} \subseteq\left(\operatorname{ker}\left(R^{2}\right)\right)^{\perp}$ $\subseteq \Im(Q)$ and that $\Im(Q)$ is invariant under $\left\{U_{t}\right\}_{t \geq 0}$. Now suppose $\mathfrak{R}$ is any subspace containing the range of $R^{2}$ and invariant under $\left\{U_{t}\right\}_{t \geq 0}$. To show that $\Im(Q) \subseteq \Re$, it suffices to show that $\Re^{\perp} \subseteq \operatorname{ker}(Q)$. But since $\Re^{\perp}$ is invariant under $\left\{U_{t}^{*}\right\}_{t \geq 0}$ and since the range of $R^{2}$ is contained in $\Re, \pi^{\perp} \subseteq \operatorname{ker}\left(R^{2}\right)$ and $R^{2} U_{t}^{*} f=0$ for all $f \in \mathfrak{R}^{\perp}$ and $t \geq 0$. So, by equation (3), $Q f$ $=\int_{0}^{\infty} U_{t} R^{2} U_{t}^{*} f d t=0$ for all $f \in \mathfrak{R}^{\perp}$, and the proof is complete.

The next theorem was first proved by Putnam in [6, Theorem 5]. There he assumed that $H$ is bounded, but later, in [7, §2.13], he proved more general results for unbounded $H$. These are slightly more general than ours, but his method of proof is considerably different from ours (cf. [2, p. 27]).

Recall that the absolutely continuous spectral subspace $\mathcal{H}_{A C}$ for $H$, or $\left\{U_{t}\right\}_{t \in \mathbf{R}}$, is the set of all vectors $f$ in $\mathcal{H}$ such that $d\|E(\lambda) f\|^{2}$ is absolutely continuous with respect to Lebesgue measure on $\mathbf{R}$ where $E$ is the spectral measure for $H$. We say $H$, or $\left\{U_{t}\right\}_{t \in \mathbf{R}}$, is absolutely continuous if $\mathcal{H}_{A C}=\mathcal{H}_{\text {. }}$ 
THEOREM II. If equation (1) has a solution, then $\mathcal{H}_{A C}$ contains the range of $R^{2}$, and so, if the smallest reducing subspace for $\left\{U_{t}\right\}_{t \in \mathbf{R}}$ containing the range of $R^{2}$ is all of $\mathcal{H}$, then $H$ is absolutely continuous.

Proof. We prove more than we need, but the excess will be used later. Let $Q$ be the principal solution to equation (1) and write $Q=C^{*} P C$ where $C$ is the bounded linear transformation from $\mathcal{H}$ to $\mathbf{L}_{\mathscr{D}}^{2}(\mathbf{R})$ constructed in the proof of Theorem I. Since $\mathfrak{s}(Q)=\mathfrak{s}(P C)$ is the smallest invariant subspace for $\left\{U_{t}\right\}_{t \geq 0}$ containing the range of $R^{2}$ by Lemma 3.1 , it is clear that since $C U_{t}^{*}=S_{t}^{*} C$ for all $t, \Im(C)$ is the smallest reducing subspace for $\left\{U_{t}\right\}_{t \in \mathbf{R}}$ containing the range of $R^{2}$. Call this subspace $\Re$, and let $C=Y W$ be the polar decomposition of $C$; i.e., $Y=\left(C C^{*}\right)^{1 / 2}$ and $W$ is the unique partial isometry with initial space $\Re$ such that $C=Y W$. Then since $S_{t}^{*} C=C U_{t}^{*}$ for all $t$, we find that $Y$ commutes with $\left\{S_{t}\right\}_{t \in \mathbf{R}}$ and that $S_{t}^{*} W=W U_{t}^{*}$ for all $t$. Hence $W$ effects a unitary equivalence between $\left\{U_{t} \mid \mathscr{\Re}\right\}_{t \in \mathbf{R}}$ and the restriction of $\left\{S_{t}\right\}_{t \in \mathbf{R}}$ to a reducing subspace $\tilde{\mathcal{T}}$ in $\mathbf{L}_{\mathscr{D}}^{2}(\mathbf{R})$. Let $\mathfrak{f}$ denote the Fourier transform regarded as a unitary operator on $\mathbf{L}_{\mathscr{D}}^{2}(\mathbf{R})$; for $t$ in $\mathbf{R}$, let $\chi_{t}$ denote the multiplication operator on $\mathbf{L}_{\mathscr{Q}}^{2}(\mathbf{R})$ determined by $e^{i t x}$; let $\mathfrak{\tau}=\mathfrak{F} \tilde{\mathscr{T}}$; and let $V=\mathfrak{F} W$. Then $\mathfrak{F} S_{t}=\chi_{t} \mathfrak{F}, \mathfrak{x}$ reduces $\left\{\chi_{t}\right\}_{t \in \mathbf{R}}$, and $V$ is a Hilbert space isomorphism from $\Re$ onto $\Re$ satisfying $V\left(U_{t} \mid \mathfrak{R}\right) V^{*}=\chi_{t} \mid \Re$ for all $t$. Since $\mathbf{L}_{\mathscr{D}}^{2}(\mathbf{R})$ is a direct integral and since $\Re$ reduces $\left\{\chi_{t}\right\}_{t \in \mathbf{R}}$ (which generates the algebra of diagonalizable operators on $\mathbf{L}_{\mathscr{\mathcal { D }}}^{2}(\mathbf{R})$ ), we may write $\Re$ as a direct integral $\Re=\int_{\mathbf{R}}^{\oplus} \Re(x) d x$ where $d x$ is Lebesgue measure on $\mathbf{R}$ and where $\Re(x)$ is a subspace of $\mathscr{D}$ for each $x$ in $\mathbf{R}$ (see [1, Chapter II]). Thus, since for each Borel set $M \subseteq \mathbf{R}, V(E(M) \mid \Re) V^{*}=I_{M} \mid \mathcal{N}$, where $I_{M}$ denotes the characteristic function of $M$, it follows that $\left\{U_{t} \mid \mathfrak{R}\right\}_{t \in \mathbf{R}}$ is absolutely continuous. This completes the proof.

We note that the closed support of the direct integral representation of $\mathscr{T}$, which is the closed support of the spectral measure for $H$, is precisely the spectrum of $H \mid \mathfrak{T}, \Lambda(H \mid \mathfrak{N})$; i.e., $\Re=\int_{\Lambda(H \mid \mathfrak{N})}^{\oplus} \mathfrak{R}(x) d x$.

THEOREM III. Let $X$ be a solution to equation (1) and suppose that the smallest reducing subspace for $H$ containing the range of $R^{2}$ is all of $\mathcal{H}$. Let $V$ be the Hilbert space isomorphism from $\mathcal{H}$ onto $\Re=\int_{\Lambda(H)}^{\oplus} \mathfrak{N}(x) d x$ constructed in the proof of Theorem II so that $V U_{t} V^{*}=\chi_{t} \mid \Re$ for all $t$. Then there are selfadjoint decomposable operators on $\Re$ given by functions $M(\cdot)$ and $K(\cdot)$ with $K(x) \geq 0$ a.e. such that

$$
\begin{aligned}
& \left(V X V^{*}\right) f(x) \\
& \quad=M(x) f(x)-\frac{i}{2 \pi} p . v . \int_{\Lambda(H)} K(x) K(y)(x-y)^{-1} f(y) d y \text { a.e. }
\end{aligned}
$$

for all $f \in \mathfrak{R}$ where p.v. denotes principal value.

Proof. We continue with the notation established in the proof of Theorem II. By Lemma 2.1 , the selfadjoint operator $A=X-Q$ commutes with $\left\{U_{t}\right\}_{t \in \mathbf{R}}$ and so $V A V^{*}$ commutes with $\left\{\chi_{t} \mid \mathcal{X}\right\}_{t \in \mathbf{R}}$. Hence $V A V^{*}$ is decomposable and therefore is given by a selfadjoint operator-valued function $L(\cdot)$ on $\Lambda(H)$. On the other hand, $V Q V^{*}=(\mathfrak{F} W)\left(W^{*} Y\right) P(Y W)\left(W^{*} \mathfrak{F}^{*}\right) \mid \mathfrak{\Re}$ $=\left(\mathfrak{F} Y \mathfrak{F}^{*}\right)\left(\mathfrak{F} P \mathfrak{F}^{*}\right)\left(\mathfrak{F} Y \mathfrak{F}^{*}\right) \mid \mathfrak{N}$. But $Y$ commutes with $\left\{S_{t}\right\}_{t \in \mathbf{R}}$, so $\mathfrak{F} Y \mathfrak{F}^{*}$ is 
decomposable, and is therefore given by an operator-valued function $K(\cdot)$ on $\Lambda(H)$. Moreover, since $Y \geq 0, K(x) \geq 0$ a.e. on $\Lambda(H)$. Finally, observe that $\mathfrak{F} P \mathfrak{F}^{*}=(I-i \mathfrak{g}) / 2$ where $\mathfrak{Q}$ is the Hilbert transform. Putting these things together, and writing $M(\cdot)=L(\cdot)+K^{2}(\cdot) / 2$, we arrive at equation (4). This completes the proof.

\section{REFERENCES}

1. J. Dixmier, Les algèbras d'opérateurs dans l'espace Hilbertien, Gauthier-Villars, Paris, 1969.

2. R. G. Douglas, On the operator equation $S^{*} X T=X$ and related topics, Acta Sci. Math. (Szeged) 30 (1969), 19-32. MR 40 \#3347.

3. - On extending commutative semigroups of isometries, Bull. London Math. Soc. 1 (1969), 157-159. MR 39 \#7458.

4. T. Kato, Smooth operators and commutators, Studia Math. 31 (1968), 535-546. MR 38 \#2631.

5. J. D. Pincus, Commutators and systems of singular integral equations. I, Acta Math. 121 (1968), 219-249. MR 39 \#2026.

6. C. R. Putnam, Commutators and absolutely continuous operators, Trans. Amer. Math. Soc. 87 (1958), 513-525. MR 20 \#6659.

7. - Commutation properties of Hilbert space operators and related topics, Ergebnisse der Mathematik und ihrer Grenzgebiete, Band 36, Springer-Verlag, New York, 1967. MR 36 \# 707.

8. Hsia Tao-hsing (Xa Dao-xeng), On non-normal operators. I, Acta Math. Sinica 12 (1962), 216-228 = Chinese Math. 3 (1963), 232-246. MR 26 \#6773.

Department of Mathematics, University of Iowa, Iowa City, Iowa 52242 